\title{
New Enantioselective Entry to Cycloheptane Amino Acid Polyols
}

Claudio Curti, Franca Zanardi, Lucia Battistini, Andrea Sartori, Gloria Rassu, Luciana Auzzas, Annamaria Roggio, Luigi Pinna, and Giovanni Casiraghi*

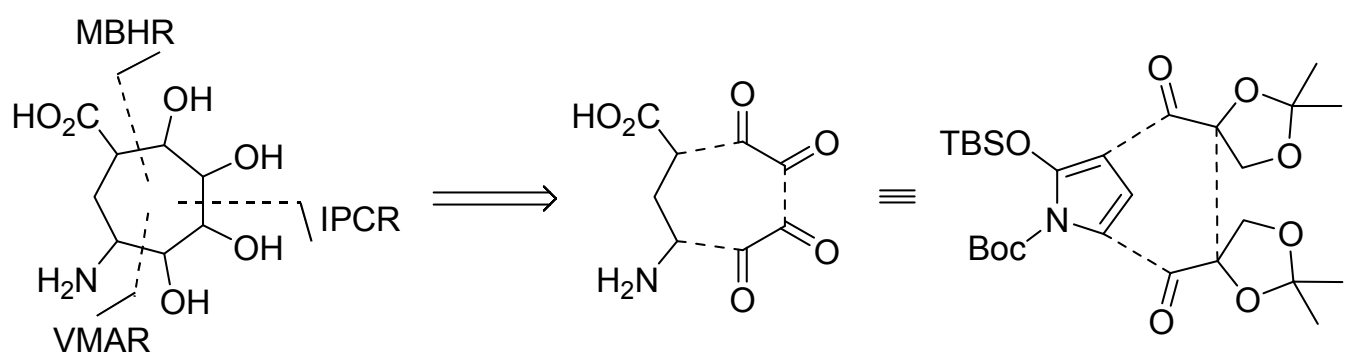

giovanni.casiraghi@unipr.it

\section{SUPPORTING INFORMATION}

Title Page and Table of Contents

General Experimental Procedures

Materials

Experimental Procedures and Spectroscopic data for compounds 4, 8-10, 14-19, 22-30

References

Copies of ${ }^{1} \mathrm{H}$ NMR spectra $\left(600 \mathrm{MHz}, \mathrm{CDCl}_{3}\right.$ or $\left.\mathrm{D}_{2} \mathrm{O}\right)$ of compounds $\mathbf{5}$, 12-14, 20-21 
General Experimental Procedures. All organic solvents were dried and freshly distilled before use according to literature procedures. All moisture sensitive reactions were carried out under a positive pressure of nitrogen or argon. TLC analysis was performed on silica gel $60 \mathrm{~F}_{254}$ plates with visualization under short-wavelength UV light or by dipping the plates with molybdate reagent (aqueous $\mathrm{H}_{2} \mathrm{SO}_{4}$ solution of cerium sulfate/ammonium molybdate) followed by heating. Flash chromatography was performed on 40-63 $\mu \mathrm{m}$ silica gel using the indicated solvent mixtures. Melting points were determined with an optical thermomicroscope and are uncorrected. Optical rotations were measured at ambient temperature using a $100-\mathrm{mm}$ cell with a $1-\mathrm{mL}$ capacity and are given in units of $10^{-1} \mathrm{deg} \mathrm{cm}^{2} \mathrm{~g}^{-1} .{ }^{1} \mathrm{H}$ and ${ }^{13} \mathrm{C}$ NMR spectra were recorded at $300 / 75 \mathrm{MHz}$ or $600 / 150 \mathrm{MHz}$. Chemical shifts $(\delta)$ are given in parts per million (ppm) using chloroform- $d\left(\mathrm{CHCl}_{3} \delta_{\mathrm{H}} 7.26, \mathrm{CDCl}_{3} \delta_{\mathrm{C}} 77.0\right)$, or deuterium oxide (DOH $\delta_{\mathrm{H}}$ $4.75)$ as internal references. High-resolution mass spectrometry (HRMS) measurements were performed on a mass spectrometer equipped with an external electrospray ion source.

Materials. $N$-(tert-Butyloxycarbonyl)-2-[(tert-butyldimethylsilyl)oxy]pyrrole (1) was prepared from pyrrole according to a described protocol. ${ }^{1}$ 2,3-O-Isopropylidene-D-glyceraldehyde $(R-2)$ was prepared from D-mannitol according to a recently optimized protocol. ${ }^{2}$ The preparation of 2,3-Oisopropylidene-L-glyceraldehyde ( $S$-2) was carried out starting from 5,6-O-isopropylidene-L-gulonic acid 1,4-lactone following a known protocol. ${ }^{3}$ A samarium (II) iodide solution in THF was prepared as follows: samarium metal powder $(0.15 \mathrm{~g}, 1.0 \mathrm{mmol})$ freshly obtained from an ingot of 99.9\% pure samarium was added under a flow of argon to an oven-dried round-bottom flask containing a magnetic stirring bar and a septum inlet. The flask and the samarium had been flame-dried and cooled under a stream of argon. Anhydrous and deoxygenated THF $(10 \mathrm{~mL})$ was added and the resulting heterogeneous mixture was vigorously stirred and cooled to $0{ }^{\circ} \mathrm{C}$. Neat diiodomethane $(228 \mathrm{mg}, 0.85 \mathrm{mmol})$ was added and the resulting dark green slurry was stirred at the same temperature for $15 \mathrm{~min}$, then allowed to warm to $25{ }^{\circ} \mathrm{C}$ and vigorously stirred for an additional hour. The persistent deep blue solution of $\mathrm{SmI}_{2}$ was titrated by a $0.1 \mathrm{M}$ solution of iodine in THF and resulted to be $0.06 \mathrm{M}$.

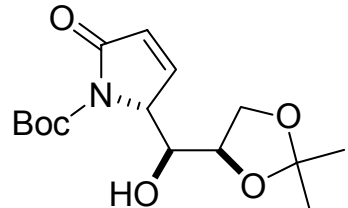

3 (1'S,2R,4"R)-2-[(2,2-Dimethyl-[1,3]dioxolan-4-yl)hydroxymethyl]-5-oxo-2,5dihydro-1H-pyrrole-1-carboxylic Acid tert-Butyl Ester (3). Typical Procedure. ${ }^{4}$ To a stirring solution of silyloxypyrrole 1 (700 $\left.\mathrm{mg}, 2.35 \mathrm{mmol}\right)$ in anhydrous $\mathrm{Et}_{2} \mathrm{O}(20 \mathrm{~mL})$ under argon atmosphere at $-90{ }^{\circ} \mathrm{C}$ was added an ether solution of aldehyde $(R)-2$ (367 $\mathrm{mg}, 2.82 \mathrm{mmol}$ dissolved in $8 \mathrm{~mL}$ of $\mathrm{Et}_{2} \mathrm{O}$ ). The resulting mixture was allowed to cool to $-90{ }^{\circ} \mathrm{C}$ for $10 \mathrm{~min}$, then $\mathrm{SnCl}_{4}\left(3.06 \mathrm{~mL}\right.$ of a $1.0 \mathrm{M}$ solution in $\left.\mathrm{CH}_{2} \mathrm{Cl}_{2}, 3.06 \mathrm{mmol}\right)$ was slowly added dropwise to the mixture at the same temperature over a period of $30 \mathrm{~min}$. After $3 \mathrm{~h}$, the reaction mixture was quenched at $-90{ }^{\circ} \mathrm{C}$ with saturated aqueous $\mathrm{NaHCO}_{3}(10 \mathrm{~mL})$ and solid 
$\mathrm{NaHCO}_{3}(1.0 \mathrm{~g})$ and temperature was allowed to reach ambient value $\left(20^{\circ} \mathrm{C}\right)$. Further portions of solid $\mathrm{NaHCO}_{3}$ were added until neutral $\mathrm{pH}$ was achieved. The mixture was concentrated under vacuum to remove the ethereal phase. The water mixture was washed with hexanes $(2 \times 15 \mathrm{~mL})$, and then extracted with EtOAc $(3 \times 15 \mathrm{~mL})$. The combined organic layers were dried $\left(\mathrm{MgSO}_{4}\right)$ and concentrated in vacuum to give a solid crude residue which was crystallized from EtOAc/hexane to give unsaturated lactam 3 (604 mg, 82\%, de> 98\%) as a white solid: mp 138-140 ${ }^{\circ} \mathrm{C} ;[\alpha]_{\mathrm{D}}{ }^{20}+197.6\left(c 0.8, \mathrm{CHCl}_{3}\right) ;{ }^{1} \mathrm{H}$ NMR $\left(300 \mathrm{MHz}, \mathrm{CDCl}_{3}\right) \delta 7.43(\mathrm{dd}, J=6.3,2.1 \mathrm{~Hz}, 1 \mathrm{H}), 6.13(\mathrm{dd}, J=6.3,1.5 \mathrm{~Hz}, 1 \mathrm{H}), 4.81(\mathrm{dt}, J=$ 5.7, 2.4 Hz, 1H), 4.09 (ddd, $J=6.0,5.7,3.9 \mathrm{~Hz}, 1 \mathrm{H}), 4.01$ (q, $J=6.0 \mathrm{~Hz}, 1 \mathrm{H}), 3.94$ (dd, $J=8.1,6.0 \mathrm{~Hz}$, $1 \mathrm{H}), 3.86(\mathrm{dd}, J=8.1,6.0 \mathrm{~Hz}, 1 \mathrm{H}), 3.63(\mathrm{~d}, J=3.9 \mathrm{~Hz}, 1 \mathrm{H}), 1.57(\mathrm{~s}, 9 \mathrm{H}), 1.37(\mathrm{~s}, 3 \mathrm{H}), 1.32(\mathrm{~s}, 3 \mathrm{H}),{ }^{13} \mathrm{C}$ NMR (75 MHz, $\left.\mathrm{CDCl}_{3}\right) \delta 168.9,150.9,148.2,126.9,109.2,83.8,75.6,72.6,66.4,65.6,28.0$ (3C), 26.4, 25.1. Anal. Calcd for $\mathrm{C}_{15} \mathrm{H}_{23} \mathrm{NO}_{6}$ : C, 57.50; H, 7.40; N, 4.47. Found: C, 57.31; H, 7.35; N, 4.32.

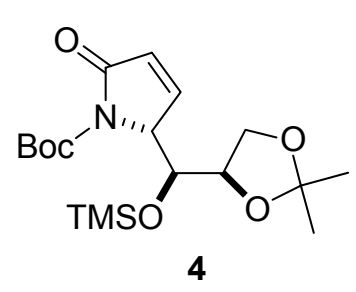

$\left(1^{\prime} S, 2 R, 4^{\prime \prime} R\right)-2-[(2,2-D i m e t h y l-[1,3]$ dioxolan-4yl)(trimethylsilanyloxy)methyl]-5-oxo-2,5-dihydro-1 $H$-pyrrole-1-carboxylic Acid tert-Butyl Ester (4). Typical Procedure. To a stirring solution of unsaturated lactam $3(604 \mathrm{mg}, 1.93 \mathrm{mmol})$ in pyridine $(5 \mathrm{~mL})$, cooled to $0{ }^{\circ} \mathrm{C}$ under argon atmosphere, trimethylsilyl chloride (TMSCl, $0.49 \mathrm{~mL}, 3.85 \mathrm{mmol}$ ) was added dropwise, and the resulting mixture was allowed to reach ambient value $\left(20^{\circ} \mathrm{C}\right)$. After 30 min, the reaction was quenched with distilled water and extracted with $\mathrm{CH}_{2} \mathrm{Cl}_{2}(3 \times 5 \mathrm{~mL})$. The combined organic layers were washed with water, dried over $\mathrm{MgSO}_{4}$, filtered, and concentrated under reduced pressure to leave a crude product which was purified by silica gel flash chromatography (EtOAc/hexanes 6:4). Protected lactam $4(729 \mathrm{mg}, 98 \%)$ was obtained as a glassy solid: $[\alpha]_{\mathrm{D}}{ }^{20}+168.9$ $\left(c\right.$ 6.2, $\left.\mathrm{CHCl}_{3}\right) ;{ }^{1} \mathrm{H}$ NMR $\left(300 \mathrm{MHz}, \mathrm{CDCl}_{3}\right) \delta 7.23(\mathrm{dd}, J=6.2,2.1 \mathrm{~Hz}, 1 \mathrm{H}), 6.12(\mathrm{dd}, J=6.2,1.7 \mathrm{~Hz}$, $1 \mathrm{H}), 4.58(\mathrm{dt}, J=4.8,1.8 \mathrm{~Hz}, 1 \mathrm{H}), 4.50(\mathrm{t}, J=5.1 \mathrm{~Hz}, 1 \mathrm{H}), 3.6-3.8(\mathrm{~m}, 3 \mathrm{H}), 1.51(\mathrm{~s}, 9 \mathrm{H}), 1.28(\mathrm{~s}, 3 \mathrm{H})$, $1.18(\mathrm{~s}, 3 \mathrm{H}), 0.16(\mathrm{~s}, 9 \mathrm{H}) ;{ }^{13} \mathrm{C} \mathrm{NMR}\left(75 \mathrm{MHz}, \mathrm{CDCl}_{3}\right) \delta 169.0,149.4,147.5,128.3,109.2,83.1,74.9$, 71.2, 66.2, 65.3, 28.3 (3C), 26.4, 25.1, 0.3 (3C). Anal. Calcd for $\mathrm{C}_{18} \mathrm{H}_{31} \mathrm{NO}_{6} \mathrm{Si}: \mathrm{C}, 56.08 ; \mathrm{H}, 8.10$; $\mathrm{N}$, 3.63. Found: C, 56.11; H, 8.16; N, 3.53.

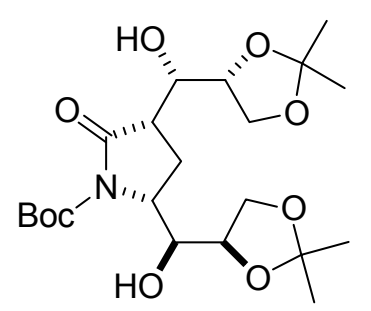

8 $\left(1^{\prime} S, 1^{\prime \prime \prime} S, 3 S, 4^{\prime \prime} R, 4^{\prime V} R, 5 R\right)-3,5-B i s[(2,2-d i m e t h y l-[1,3]$ dioxolan-4yl)hydroxymethyl]-2-oxopyrrolidine-1-carboxylic Acid tert-Butyl Ester (8). Typical Procedure. To a solution of lactam 7 (280 mg, $0.54 \mathrm{mmol})$ in methanol $(15 \mathrm{~mL})$, solid citric acid $(311 \mathrm{mg}, 1.62 \mathrm{mmol})$ was added and the resulting 
mixture was allowed to stir at room temperature for $6 \mathrm{~h}$, while further portions of citric acid $(6 \times 311$ $\mathrm{mg}, 9.72 \mathrm{mmol}$ ) were added. The reaction mixture was then diluted with water and treated with saturated aqueous $\mathrm{NaHCO}_{3}$ solution. Extraction with EtOAc $(3 \times 10 \mathrm{~mL})$, drying over $\mathrm{MgSO}_{4}$ and concentration under reduced pressure afforded a crude residue which was purified by silica gel flash chromatography (EtOAc/hexanes 75:25). Pure diol 8 was obtained (228 mg, 95\%) as a white solid: mp $125-130{ }^{\circ} \mathrm{C} ;[\alpha]_{\mathrm{D}}{ }^{20}+10.2\left(c 1.0, \mathrm{CHCl}_{3}\right) ;{ }^{1} \mathrm{H} \mathrm{NMR}\left(300 \mathrm{MHz}, \mathrm{CDCl}_{3}\right) \delta 4.89$ (bs, $\left.1 \mathrm{H}, \mathrm{OH}\right), 4.37$ (ddd, $J=9.6,5.0,2.5 \mathrm{~Hz}, 1 \mathrm{H}, \mathrm{H} 5), 4.35$ (bs, 1H, OH), 4.0-4.2 (m, 4H, H5"a, H1', H5 $\left.{ }^{\prime N} \mathrm{a}, \mathrm{H} 5^{\prime \prime} \mathrm{b}\right), 3.99$ (m, 1H, $\mathrm{H} 4^{\prime \prime}$ ), 3.90 (m, 2H, H5"b, H4"), 3.73 (bt, $\left.J=6.1 \mathrm{~Hz}, 1 \mathrm{H}, \mathrm{H} 1^{\prime \prime \prime}\right), 2.98$ (ddd, $J=12.1,4.4,1.5 \mathrm{~Hz}, 1 \mathrm{H}, \mathrm{H} 3$ ), 2.32 (ddd, $J=13.8,12.4,9.9 \mathrm{~Hz}, 1 \mathrm{H}, \mathrm{H} 4 \mathrm{a}), 2.03$ (ddd, $J=13.9,4.4,2.8 \mathrm{~Hz}, 1 \mathrm{H}, \mathrm{H} 4 \mathrm{~b}$ ), 1.47 (s, 9H, Boc), 1.41 (s, 3H, Me), 1.39 (s, 3H, Me), 1.33 (s, 3H, Me), 1.32 (s, 3H, Me); ${ }^{13} \mathrm{C}$ NMR (75 MHz, $\left.\mathrm{CDCl}_{3}\right) \delta 177.2(\mathrm{C} 2), 150.8$ (Boc), $109.6\left(\mathrm{CMe}_{2}\right), 109.3\left(\mathrm{CMe}_{2}\right), 83.6$ (Boc), 76.9, 76.4, 76.3, 70.9 (C4", $\left.\mathrm{C}^{\prime}, \mathrm{C}^{\prime \prime}, \mathrm{C} 1{ }^{\prime \prime \prime}\right), 67.5,67.4\left(\mathrm{C}^{\prime \prime}, \mathrm{C}^{\prime \prime}\right), 58.0(\mathrm{C} 5), 45.6$ (C3), 27.8 (3C, Boc), $26.9(\mathrm{Me}), 26.7$ (Me), 25.4 (Me), $25.3(\mathrm{Me}), 21.2$ (C4). Anal. Calcd for $\mathrm{C}_{21} \mathrm{H}_{35} \mathrm{NO}_{9}$ : C, 56.62; H, 7.92; N, 3.14. Found: C, 56.74; $\mathrm{H}$, $8.02 ; \mathrm{N}, 2.99$.

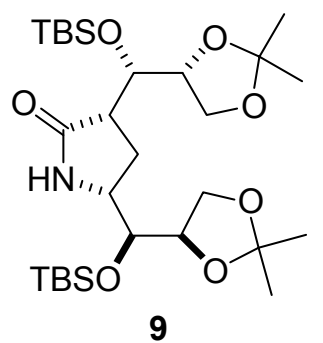

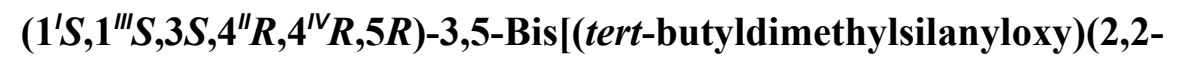
dimethyl-[1,3]dioxolan-4-yl)methyl]pyrrolidin-2-one (9). Typical Procedure. Deprotected lactam 8 (228 mg, $0.51 \mathrm{mmol})$ was dissolved in anhydrous $\mathrm{CH}_{2} \mathrm{Cl}_{2}(10$ $\mathrm{mL})$ at ambient temperature under argon and 2,6-lutidine $(365 \mu \mathrm{L}, 3.06 \mathrm{mmol})$ and tert-butyldimethylsilyl trifluoromethanesulfonate (TBSOTf, $234 \mu \mathrm{L}, 1.0 \mathrm{mmol}$ ) were sequentially added under stirring. After $2 \mathrm{~h}$, the reaction mixture was quenched with saturated $\mathrm{NH}_{4} \mathrm{Cl}$ solution, diluted with distilled water and extracted with $\mathrm{CH}_{2} \mathrm{Cl}_{2}(3 \times 10 \mathrm{~mL})$. The combined organic layers were dried over $\mathrm{MgSO}_{4}$, filtered, and concentrated under reduced pressure to furnish a crude product which was dissolved in $30 \mathrm{~mL}$ of acetonitrile, warmed to $80^{\circ} \mathrm{C}$, and the resulting mixture was treated with ammonium cerium (IV) nitrate (CAN, $56 \mathrm{mg}, 0.10 \mathrm{mmol}$ ). After $3 \mathrm{~h}$, the reaction was quenched with $10 \%$ aqueous $\mathrm{Na}_{2} \mathrm{~S}_{2} \mathrm{O}_{3}$ solution and extracted with EtOAc $(3 \times 20 \mathrm{~mL})$. The combined organic layers were dried $\left(\mathrm{MgSO}_{4}\right)$, filtered and concentrated under reduced pressure furnishing a crude residue which was purified by silica gel flash chromatography (EtOAc/hexanes 1:1). $N$-deprotected lactam 9 (234 mg, 80\%) was recovered as a white solid: mp 108-112 ${ }^{\circ} \mathrm{C} ;[\alpha]_{\mathrm{D}}{ }^{20}-4.8\left(\mathrm{c} \mathrm{5.3}, \mathrm{CHCl}_{3}\right) ;{ }^{1} \mathrm{H}$ NMR (600 MHz, $\left.\mathrm{CDCl}_{3}\right) \delta 5.63(\mathrm{~s}, 1 \mathrm{H}), 4.25(\mathrm{~d}, J=7.8 \mathrm{~Hz}, 1 \mathrm{H}), 4.09(\mathrm{~m}, 2 \mathrm{H}), 3.92(\mathrm{~m}, 2 \mathrm{H}), 3.83(\mathrm{~m}$, 2H), $3.70(\mathrm{dd}, J=8.4,3.6 \mathrm{~Hz}, 1 \mathrm{H}), 3.43(\mathrm{td}, J=9.0,6.6 \mathrm{~Hz}, 1 \mathrm{H}), 2.87(\mathrm{dd}, J=10.2,9.6 \mathrm{~Hz}, 1 \mathrm{H}), 2.11$ (ddd, $J=12.6,8.4,7.2 \mathrm{~Hz}, 1 \mathrm{H}), 2.05$ (td, $J=12.0,10.2 \mathrm{~Hz}, 1 \mathrm{H}), 1.45(\mathrm{~s}, 3 \mathrm{H}), 1.42(\mathrm{~s}, 3 \mathrm{H}), 1.37$ (s, 3H), $1.35(\mathrm{~s}, 3 \mathrm{H}), 0.93(\mathrm{~s}, 9 \mathrm{H}), 0.88(\mathrm{~s}, 9 \mathrm{H}), 0.15(\mathrm{~s}, 9 \mathrm{H}), 0.09(\mathrm{~s}, 3 \mathrm{H}) ;{ }^{13} \mathrm{C} \mathrm{NMR}\left(75 \mathrm{MHz}, \mathrm{CDCl}_{3}\right) \delta 176.8$, 109.3, 109.0, 77.3, 76.3, 76.0, 70.8, 67.9, 64.6, 55.9, 45.3, 26.7, 26.4, 26.0 (3C), 25.9 (3C), 25.4, 25.1, 
23.5, 18.3, 18.4, -3.9 (2C), -4.0, -4.9. Anal. Calcd for $\mathrm{C}_{28} \mathrm{H}_{55} \mathrm{NO}_{7} \mathrm{Si}_{2}$ : C, 58.60; H, 9.66; N, 2.44 . Found: C, 58.54; H, 9.59; N, 2.35 .

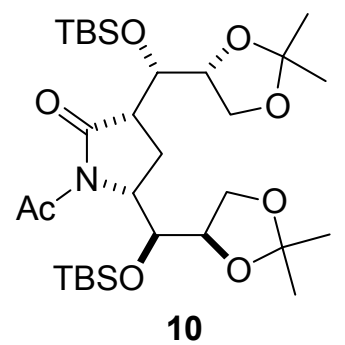

\section{$\left(1^{\prime} S, 1^{\prime \prime \prime} S, 3 S, 4^{\prime \prime} R, 4^{\prime \prime} R, 5 R\right)-1-A c e t y l-3,5-b i s[(t e r t-$}

butyldimethylsilanyloxy)(2,2-dimethyl-[1,3]dioxolan-4-yl)methyl]pyrrolidin2-one (10). Typical Procedure. To a stirring solution of lactam 9 (234 mg, 0.41 $\mathrm{mmol})$ in acetonitrile $(20 \mathrm{~mL})$ under argon, $\mathrm{Et}_{3} \mathrm{~N}(114 \mu \mathrm{L}, 0.82 \mathrm{mmol})$, acetic anhydride (39 $\mu \mathrm{L}, 0.41 \mathrm{mmol})$, and 4-dimethylaminopyridine (DMAP, $100 \mathrm{mg}$,

$0.82 \mathrm{mmol}$ ) were sequentially added. The resulting mixture was warmed to $70{ }^{\circ} \mathrm{C}$ and further portions of $\mathrm{Et}_{3} \mathrm{~N}(3 \times 114 \mu \mathrm{L}, 2.46 \mathrm{mmol})$, acetic anhydride $(3 \times 39 \mu \mathrm{L}, 1.23 \mathrm{mmol})$, and DMAP $(3 \times 100 \mathrm{mg}$, $2.46 \mathrm{mmol}$ ) were added. After $24 \mathrm{~h}$, the reaction mixture was quenched with saturated $\mathrm{NH}_{4} \mathrm{Cl}$ solution, diluted with distilled water and extracted with $\mathrm{CH}_{2} \mathrm{Cl}_{2}(3 \times 10 \mathrm{~mL})$. The combined organic layers were dried over $\mathrm{MgSO}_{4}$, filtered, and concentrated under vacuum. After flash chromatographic purification (EtOAc/hexanes 1:4) pure acetylated lactam 10 was recovered $(225 \mathrm{mg}, 89 \%)$ as a glassy solid: ${ }^{1} \mathrm{H}$ NMR (600 MHz, $\left.\mathrm{CDCl}_{3}\right) \delta 4.62\left(\mathrm{dd}, J=8.4,4.8 \mathrm{~Hz}, 1 \mathrm{H}, \mathrm{H} 1^{\prime \prime \prime}\right), 4.30$ (m, 2H, H1', H5), 4.10 (m, 2H, H5"a, H4 $\left.{ }^{\prime V}\right), 4.02\left(\mathrm{dd}, J=9.0,6.6 \mathrm{~Hz}, 1 \mathrm{H}, \mathrm{H} 5^{\prime \prime} \mathrm{a}\right), 3.95$ (dt, $\left.J=7.8,5.4 \mathrm{~Hz}, 1 \mathrm{H}, \mathrm{H} 4^{\prime \prime}\right), 3.91$ (m, 2H, H5"b, H5 $\left.{ }^{\prime v} \mathrm{~b}\right), 2.93$ (ddd, $J=11.4,9.6,1.2 \mathrm{~Hz}, 1 \mathrm{H}, \mathrm{H} 3$ ), 2.47 (s, 3H, Ac), 2.16 (td, $J=12.6,10.2 \mathrm{~Hz}, 1 \mathrm{H}, \mathrm{H} 4 \mathrm{a}$ ), 2.02 (ddd, $J=13.8,10.2,8.4 \mathrm{~Hz}, 1 \mathrm{H}, \mathrm{H} 4 \mathrm{~b}), 1.44$ (s, 3H, Me), 1.37(s, 3H, Me), 1.31 (s, 3H, Me), 1.30 (s, 3H, Me), 0.93 (s, 9H, TBS), 0.91 (s, 9H, TBS), 0.21 (s, 3H, TBS), 0.19 (s, 3H, TBS), 0.18 (s, 3H, TBS), 0.08 (s, 3H, TBS); ${ }^{13} \mathrm{C}$ NMR (150 MHz, $\left.\mathrm{CDCl}_{3}\right) \delta 176.2$ (C2), 173.0 (Ac), $109.5\left(\mathrm{CMe}_{2}\right), 109.3\left(C \mathrm{Me}_{2}\right)$,

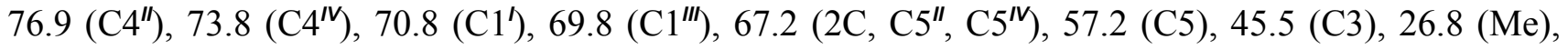
26.0 (Me), 25.9 (3C, TBS), 25.8 (Ac), 25.7 (3C, TBS), 25.6 (Me), 25.2 (Me), 18.3 (TBS), 17.9 (TBS), 16.4 (C4), -3.7 (TBS), -4.2 (TBS), -4.9 (TBS), -5.2 (TBS). Anal. Calcd for $\mathrm{C}_{30} \mathrm{H}_{57} \mathrm{NO}_{8} \mathrm{Si}_{2}: \mathrm{C}, 58.50$; H, 9.33; N, 2.27. Found: C, 58.44; H, 9.25; N, 2.20.

$\left(1^{\prime} R, 1^{\prime \prime \prime} S, 4^{\prime \prime} S, 4^{\prime V} R, 5 R\right)-3-[(2,2-D i m e t h y l-[1,3]$ dioxolan-4-yl)hydroxymethyl]-5-[(2,2-dimethyl[1,3]dioxolan-4-yl)(trimethylsilanyloxy)methyl]-2-oxo-2,5-dihydro-1H-pyrrole-1-carboxylic Acid tert-Butyl Ester (14) and $\quad\left(1^{\prime} S, 1^{\prime \prime} S, 4^{\prime \prime} S, 4^{\prime \prime} R, 5 R\right)-3-[(2,2-D i m e t h y l-[1,3] d i o x o l a n-4-$ yl)hydroxymethyl]-5-[(2,2-dimethyl-[1,3]dioxolan-4-yl)(trimethylsilanyloxy)methyl]-2-oxo-2,5dihydro-1H-pyrrole-1-carboxylic Acid tert-Butyl Ester (15). The title compounds were prepared from butenolide 4 (364 mg, $0.94 \mathrm{mmol})$ and L-gliceraldehyde $(S)-2$ (245 $\mathrm{mg}, 1.88 \mathrm{mmol})$ according to the typical procedure described for $\mathbf{5}$ and $\mathbf{6}$. After flash chromatographic purification (EtOAc/hexanes $35: 65)$, adducts 14 (300 mg, 62\%) and 15 (78 mg, 16\%) were recovered in 78\% combined yield. 


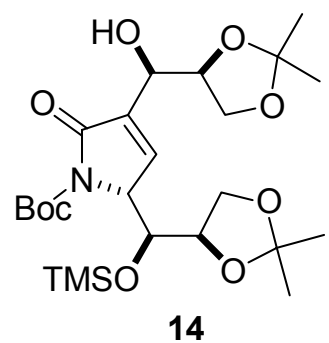

Compound 14: a colorless oil, $[\alpha]_{\mathrm{D}}^{20}+75.3\left(\right.$ c 2.2, $\left.\mathrm{CHCl}_{3}\right) ;{ }^{1} \mathrm{H}$ NMR $(600$ $\left.\mathrm{MHz}, \mathrm{CDCl}_{3}\right) \delta 7.28(\mathrm{dd}, J=2.4,1.8 \mathrm{~Hz}, 1 \mathrm{H}, \mathrm{H} 4), 4.64(\mathrm{ddt}, J=5.4,4.2,1.8 \mathrm{~Hz}$, $\left.1 \mathrm{H}, \mathrm{H} 1^{\prime}\right), 4.62$ (dt, $\left.J=4.2,1.8 \mathrm{~Hz}, 1 \mathrm{H}, \mathrm{H} 5\right), 4.58$ (bq, $\left.J=6.0 \mathrm{~Hz}, 1 \mathrm{H}, \mathrm{H} 4^{\prime \prime}\right), 4.48$ (dd, $\left.J=7.2,4.2 \mathrm{~Hz}, 1 \mathrm{H}, \mathrm{H1}{ }^{\prime \prime \prime}\right), 3.99$ (dd, $\left.J=7.8,6.6 \mathrm{~Hz}, 1 \mathrm{H}, \mathrm{H} 5{ }^{\prime \prime} \mathrm{a}\right), 3.94$ (dd, $J=$ 8.4, $6.0 \mathrm{~Hz}, 1 \mathrm{H}, \mathrm{H} 5$ "b), $3.92\left(\mathrm{dd}, J=8.4,6.6 \mathrm{~Hz}, 1 \mathrm{H}, \mathrm{H} 5^{\prime v} \mathrm{a}\right), 3.75(\mathrm{dd}, J=7.8,6.0$ $\left.\mathrm{Hz}, 1 \mathrm{H}, \mathrm{H}^{\prime v} \mathrm{~b}\right), 3.71$ (dt, $J=7.2,6.0 \mathrm{~Hz}, 1 \mathrm{H}, \mathrm{H} 4^{\prime v}$ ), 3.07 (d, J=4.2 Hz, 1H, OH), 1.60 (s, 9H, Boc), 1.49 (s, 3H, Me), 1.39 (s, 3H, Me), 1.36 (s, 3H, Me), 1.27 (s, 3H, Me), 0.25 (s, 9H, TMS); ${ }^{13} \mathrm{C}$ NMR (75 $\left.\mathrm{MHz}, \mathrm{CDCl}_{3}\right) \delta 168.2$ (C2), 149.4 (Boc), 142.0 (C4), 138.3 (C3), $109.7\left(\mathrm{CMe}_{2}\right), 109.5\left(C \mathrm{Me}_{2}\right), 83.2$

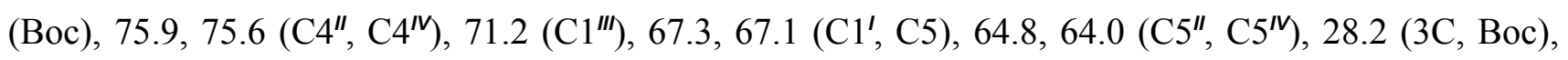
$25.2(\mathrm{Me}), 25.1(\mathrm{Me}), 25.0(\mathrm{Me}), 24.9(\mathrm{Me}), 0.2$ (3C, TMS). Anal. Calcd for $\mathrm{C}_{24} \mathrm{H}_{41} \mathrm{NO}_{9} \mathrm{Si}$ : C, 55.90; H, 8.01; N, 2.72. Found: C, 55.78; H, 8.07; N, 2.84.

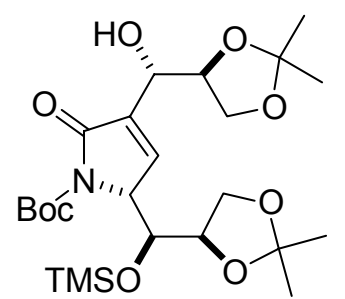

15

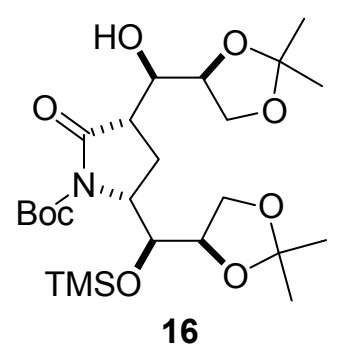

Compound 15: a colorless oil, $[\alpha]_{\mathrm{D}}{ }^{20}+70.8\left(c\right.$ 1.5, $\left.\mathrm{CHCl}_{3}\right) ;{ }^{1} \mathrm{H}$ NMR $(300$ $\left.\mathrm{MHz}, \mathrm{CDCl}_{3}\right) \delta 7.22$ (bs, 1H), $4.58(\mathrm{~m}, 1 \mathrm{H}), 4.46(\mathrm{~m}, 3 \mathrm{H}), 4.0-4.2(\mathrm{~m}, 3 \mathrm{H}), 3.92$ (m, 1H), $3.71(\mathrm{~m}, 1 \mathrm{H}), 2.98(\mathrm{bs}, 1 \mathrm{H}), 1.56(\mathrm{~s}, 9 \mathrm{H}), 1.48(\mathrm{~s}, 3 \mathrm{H}), 1.43(\mathrm{~s}, 3 \mathrm{H}), 1.32$ $(\mathrm{s}, 3 \mathrm{H}), 1.23(\mathrm{~s}, 3 \mathrm{H}), 0.2(\mathrm{~s}, 9 \mathrm{H})$. Anal. Calcd for $\mathrm{C}_{24} \mathrm{H}_{41} \mathrm{NO}_{9} \mathrm{Si}: \mathrm{C}, 55.90 ; \mathrm{H}, 8.01$; N, 2.72. Found: C, 56.01; H, 8.05; N, 2.62.

\section{$\left(1^{\prime} R, 1^{\prime \prime \prime} S, 3 S, 4^{\prime \prime} S, 4^{\prime V} R, 5 R\right)-3-[(2,2-D i m e t h y l-[1,3]$ dioxolan-4-} yl)hydroxylmethyl]-5-[(2,2-dimethyl-[1,3]dioxolan-4yl)(trimethylsilanyloxy)methyl]-2-oxopyrrolidine-1-carboxylic Acid tert-Butyl Ester (16). The title compound was prepared from 14 (300 mg, $0.58 \mathrm{mmol}$ ) according to the typical procedure described for lactam 7. After flash chromatographic purification (EtOAc/hexanes 6:4), compound $\mathbf{1 6}$ was obtained (294 $\mathrm{mg}, 98 \%$ ) as a glassy solid: $[\alpha]_{\mathrm{D}}{ }^{20}+25.7\left(c 1.0, \mathrm{CHCl}_{3}\right) ;{ }^{1} \mathrm{H}$ NMR $\left(600 \mathrm{MHz}, \mathrm{CDCl}_{3}\right) \delta 4.27(\mathrm{dd}, J=7.2,4.2 \mathrm{~Hz}, 1 \mathrm{H}$, $\left.\mathrm{H} 1^{\prime \prime \prime}\right), 4.24(\mathrm{~d}, J=3.6 \mathrm{~Hz}, 1 \mathrm{H}, \mathrm{OH}), 4.18\left(\mathrm{dt}, J=7.8,6.0 \mathrm{~Hz}, 1 \mathrm{H}, \mathrm{H} 4^{\prime \prime}\right), 4.15(\mathrm{ddd}, J=9.0,6.6,4.2 \mathrm{~Hz}$, 1H, H5), 4.11 (dd, $\left.J=9.0,6.0 \mathrm{~Hz}, 1 \mathrm{H}, \mathrm{H} 5{ }^{\prime \prime} \mathrm{a}\right), 4.09$ (q, $J=7.2 \mathrm{~Hz}, 1 \mathrm{H}, \mathrm{H} 4^{\prime \prime}$ ), 4.03 (dd, $J=7.8,6.0 \mathrm{~Hz}$, $\left.1 \mathrm{H}, \mathrm{H} 5^{\prime v} \mathrm{a}\right), 4.01\left(\mathrm{dd}, J=9.0,5.4 \mathrm{~Hz}, 1 \mathrm{H}, \mathrm{H} 5^{\prime \prime} \mathrm{b}\right), 3.74\left(\mathrm{dd}, J=7.8,6.6 \mathrm{~Hz}, 1 \mathrm{H}, \mathrm{H} 5^{\prime v} \mathrm{~b}\right), 3.69$ (td, $J=7.2$, $\left.3.0 \mathrm{~Hz}, 1 \mathrm{H}, \mathrm{H} 1^{\prime}\right), 2.72(\mathrm{ddd}, J=10.8,9.0,7.2 \mathrm{~Hz}, 1 \mathrm{H}, \mathrm{H} 3), 2.25$ (ddd, $\left.J=14.4,11.4,9.0 \mathrm{~Hz}, 1 \mathrm{H}, \mathrm{H} 4 \mathrm{a}\right)$, 2.02 (ddd, $J=14.4,9.0,6.6 \mathrm{~Hz}, 1 \mathrm{H}, \mathrm{H} 4 \mathrm{~b}), 1.53$ (s, 9H, Boc), 1.40 (s, 3H, Me), 1.34 (s, 3H, Me), 1.32 (s, $3 \mathrm{H}, \mathrm{Me}), 1.28$ (s, 3H, Me), 0.15 (s, 9H, TMS); $\left.{ }^{13} \mathrm{C} \mathrm{NMR} \mathrm{(75} \mathrm{MHz,} \mathrm{CDCl}_{3}\right) \delta 176.1$ (C2), 149.8 (Boc), $109.5\left(\mathrm{CMe}_{2}\right), 109.4\left(\mathrm{CMe}_{2}\right), 83.2$ (Boc), 77.9, 74.7, 73.6, $71.8\left(\mathrm{C}^{\prime \prime}, \mathrm{Cl}^{\prime}, \mathrm{C}^{\prime \prime}, \mathrm{C} 1^{\prime \prime \prime}\right), 67.7,67.2\left(\mathrm{C}^{\prime \prime}\right.$, $\mathrm{C}^{\prime v}$ ), 58.4 (C5), 45.0 (C3), 28.1 (3C, Boc), $26.6(\mathrm{Me}), 26.3(\mathrm{Me}), 25.3(\mathrm{Me}), 25.2(\mathrm{Me}), 20.8(\mathrm{C} 4), 0.3$ (3C, TMS). Anal. Calcd for $\mathrm{C}_{24} \mathrm{H}_{43} \mathrm{NO}_{9} \mathrm{Si}$ : C, 55.68; H, 8.37; N, 2.71. Found: C, 55.83; H, 8.21; N, 2.62. 


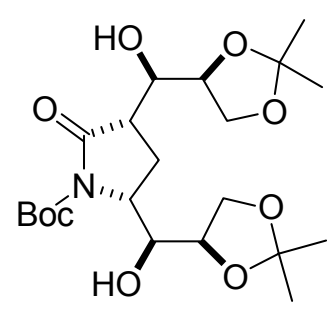

17

$\left(1^{\prime} R, 1^{\prime \prime \prime} S, 3 S, 4^{\prime \prime} S, 4^{\prime V} R, 5 R\right)-3,5-B i s[(2,2-d i m e t h y l-[1,3]$ dioxolan-4yl)hydroxymethyl]-2-oxopyrrolidine-1-carboxylic Acid tert-Butyl Ester (17). The title compound was prepared from lactam 16 (294 mg, $0.57 \mathrm{mmol})$ according to the procedure described for 8 . After flash chromatographic purification (EtOAc/hexanes 7:3), lactam 17 was obtained (229 mg, 90\%) as a light white solid: $[\alpha]_{\mathrm{D}}{ }^{20}+23.7\left(c\right.$ 1.2, $\left.\mathrm{CHCl}_{3}\right) ;{ }^{1} \mathrm{H}$ NMR $\left(300 \mathrm{MHz}, \mathrm{CDCl}_{3}\right) \delta 4.2-4.4(\mathrm{~m}, 3 \mathrm{H})$, 4.0-4.2 (m, 3H), 3.9-4.0 (m, 3H), $3.78(\mathrm{~m}, 1 \mathrm{H}), 3.70(\mathrm{dd}, J=7.2,4.8 \mathrm{~Hz}, 1 \mathrm{H}), 2.88(\mathrm{dt}, J=11.1,5.7 \mathrm{~Hz}$, 1H), 2.49 (ddd, $J=14.1,11.4,9.3 \mathrm{~Hz}, 1 \mathrm{H}), 2.06$ (ddd, $J=14.1,6.0,4.8 \mathrm{~Hz}, 1 \mathrm{H}), 1.54$ (s, 9H), 1.41 (s, $3 \mathrm{H}), 1.40(\mathrm{~s}, 3 \mathrm{H}), 1.36(\mathrm{~s}, 3 \mathrm{H}), 1.35(\mathrm{~s}, 3 \mathrm{H}) ;{ }^{13} \mathrm{C} \mathrm{NMR}\left(75 \mathrm{MHz}, \mathrm{CDCl}_{3}\right) \delta 175.3,151.4,109.4,109.3$, 83.8, 76.7, 76.6, 75.6, 74.4, 67.3, 67.2, 58.6, 45.1, 27.9 (3C), 26.7, 26.5, 25.3, 25.2, 25.1. Anal. Calcd for $\mathrm{C}_{21} \mathrm{H}_{35} \mathrm{NO}_{9}$ : C, 56.62; H, 7.92; N, 3.14. Found: C, 56.70; H, 7.99; N, 3.02 .

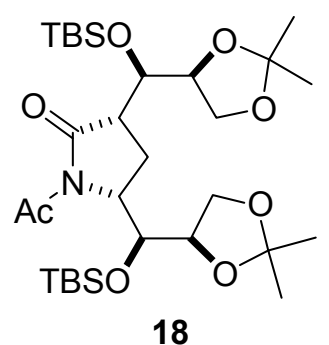

\section{$\left(1^{\prime} R, 1^{\prime \prime \prime} S, 3 S, 4^{\prime \prime} S, 4^{\prime V} R, 5 R\right)-1-A c e t y l-3,5-b i s[(t e r t-$}

butyldimethylsilanyloxy)(2,2-dimethyl-[1,3]dioxolan-4-yl)methyl]pyrrolidin2-one (18). The title compound was prepared from 17 (229 $\mathrm{mg}, 0.51 \mathrm{mmol})$ by adopting the two-step typical procedure to 10. After flash chromatographic purification (EtOAc/hexanes 1:4), protected lactam 18 was recovered (242 mg, $77 \%)$ as a glassy solid: $[\alpha]_{578}{ }^{20}+26.0\left(\right.$ c $\left.1.0, \mathrm{CHCl}_{3}\right) ;{ }^{1} \mathrm{H} \mathrm{NMR}\left(300 \mathrm{MHz}, \mathrm{CDCl}_{3}\right)$ $\delta 4.63(\mathrm{dd}, J=7.5,4.8 \mathrm{~Hz}, 1 \mathrm{H}), 4.1-4.3(\mathrm{~m}, 5 \mathrm{H}), 4.02(\mathrm{dd}, J=8.3,6.5 \mathrm{~Hz}, 1 \mathrm{H}), 3.92(\mathrm{~m}, 1 \mathrm{H}), 3.89$ (dd, $J=8.3,6.6 \mathrm{~Hz}, 1 \mathrm{H}), 2.73(\mathrm{ddd}, J=12.0,10.2,2.4 \mathrm{~Hz}, 1 \mathrm{H}), 2.43(\mathrm{~s}, 3 \mathrm{H}), 2.04(\mathrm{~m}, 2 \mathrm{H}), 1.38(\mathrm{~s}, 3 \mathrm{H})$, $1.32(\mathrm{~s}, 6 \mathrm{H}), 1.29(\mathrm{~s}, 3 \mathrm{H}), 0.92(\mathrm{~s}, 9 \mathrm{H}), 0.89(\mathrm{~s}, 9 \mathrm{H}), 0.18(\mathrm{~s}, 3 \mathrm{H}), 0.16(\mathrm{~s}, 3 \mathrm{H}), 0.14(\mathrm{~s}, 3 \mathrm{H}), 0.13(\mathrm{~s}$, $3 \mathrm{H}) ;{ }^{13} \mathrm{C}$ NMR $\left(75 \mathrm{MHz}, \mathrm{CDCl}_{3}\right) \delta 174.5,173.1,109.7,109.1,75.9,74.1,72.9,70.1,67.9,67.0,56.9$, 47.8, 26.0, 25.9, 25.8 (2C), 25.7 (3C), 25.6 (3C), 25.1, 18.5, 18.0, 17.9, -4.3, -4.4, -4.6, -4.9. Anal. Calcd for $\mathrm{C}_{30} \mathrm{H}_{57} \mathrm{NO}_{8} \mathrm{Si}_{2}:$ C, 58.50; H, 9.33; N, 2.27. Found: C, 58.37; H, 9.39; N,<smiles>CN1C(=O)[C@@H]2C(C(C=O)OC(F)(F)F)CC(C(C=O)OC(C)(C)C)C21</smiles>

19 2.38 .

\section{$\left(2 S, 2^{\prime} R, 2^{\prime \prime} R, 4^{\prime \prime} S\right)-2,2^{\prime}-(1-A c e t y l-5-0 x 0 p y r r o l i d i n e-2,4-d i y l) b i s(t e r t-$}

butyldimethylsilanyloxyacetaldehyde) (19). The title compound was prepared from lactam 18 (242 mg, $0.39 \mathrm{mmol})$ according to the typical procedure described for compound 11. Aldehyde 19 was obtained (170 mg, 94\%) as a colorless oil: $[\alpha]_{578}{ }^{20}$ +52.0 (c 1.0, $\left.\mathrm{CHCl}_{3}\right) ;{ }^{1} \mathrm{H}$ NMR (300 MHz, $\left.\mathrm{CDCl}_{3}\right) \delta 9.86(\mathrm{~s}, 1 \mathrm{H}, \mathrm{H} 1), 9.52$ (s, 1H, H1) 4.27 (m, 3H, H2', H2, H2"), 3.17 (ddd, $J=12.6,9.0,3.5 \mathrm{~Hz}, 1 \mathrm{H}, \mathrm{H} 4^{\prime \prime}$ ), 2.50 (s, 3H, Ac), 2.28 (ddd, $J=12.6,9.0,6.9$ Hz, 1H, H3"a), 1.90 (td, $J=12.4,10.2 \mathrm{~Hz}, 1 \mathrm{H}, \mathrm{H3}$ "b), 0.95 (s, 9H, TBS), 0.93 (s, 9H, TBS), 0.14 (s, 3H, TBS), 0.13 (s, 3H, TBS), 0.11 (s, 6H, TBS); ${ }^{13} \mathrm{C}$ NMR (75 MHz, $\left.\mathrm{CDCl}_{3}\right) \delta 203.5,201.6,173.4,172.4$, 
75.5 (2C), 57.9, 48.2, 25.8 (3C), 25.6 (3C), 25.2, 23.6, 18.2, 17.9, -4.5, -4.7, -4.8, -4.9. Anal. Calcd for $\mathrm{C}_{22} \mathrm{H}_{41} \mathrm{NO}_{6} \mathrm{Si}_{2}: \mathrm{C}, 56.01 ; \mathrm{H}, 8.76 ; \mathrm{N}, 2.97$. Found: $\mathrm{C}, 55.93 ; \mathrm{H}, 8.65 ; \mathrm{N}, 3.08$.

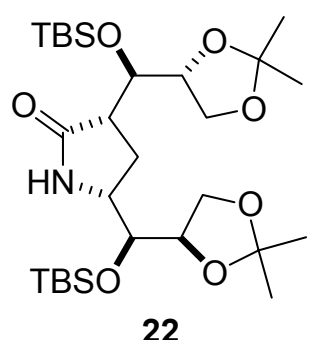

22

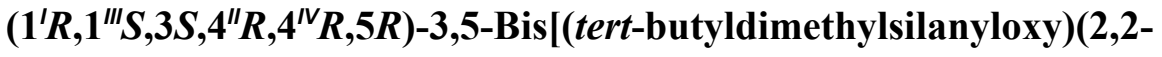

dimethyl-[1,3]dioxolan-4-yl)methyl]pyrrolidin-2-one (22). The title compound was prepared from $6(92 \mathrm{mg}, 0.18 \mathrm{mmol})$ by adopting the three-step typical procedure described for 9. After flash chromatographic purification (EtOAc/hexanes 1:1), lactam 22 was recovered (91 mg, 88\%) as a glassy solid: ${ }^{1} \mathrm{H}$ $\operatorname{NMR}\left(600 \mathrm{MHz}, \mathrm{CDCl}_{3}\right) \delta 5.69(\mathrm{bs}, 1 \mathrm{H}, \mathrm{NH}), 4.54\left(\mathrm{dt}, J=7.8,6.0 \mathrm{~Hz}, 1 \mathrm{H}, \mathrm{H} 4^{\prime \prime}\right)$, $4.07\left(\mathrm{dd}, J=7.8,6.6 \mathrm{~Hz}, 1 \mathrm{H}, \mathrm{H} 5^{\prime \prime} \mathrm{a}\right), 4.05\left(\mathrm{~m}, 1 \mathrm{H}, \mathrm{H} 4^{\prime v}\right), 3.98\left(\mathrm{t}, J=7.2 \mathrm{~Hz}, 1 \mathrm{H}, \mathrm{H} 5^{\prime v} \mathrm{a}\right), 3.93(\mathrm{dd}, J=$ 5.4, 3.6 Hz, 1H, H1'), 3.85 (t, $\left.J=7.8 \mathrm{~Hz}, 1 \mathrm{H}, \mathrm{H} 5^{\prime \prime} \mathrm{b}\right), 3.78$ (dd, $\left.J=8.4,4.2 \mathrm{~Hz}, 1 \mathrm{H}, \mathrm{H} 1^{\prime \prime \prime}\right), 3.68$ (t, $J=7.8$ Hz, 1H, H5"b), 3.46 (q, $J=8.4$ Hz, 1H, H5), 2.64 (td, $J=9.6,3.6$ Hz, 1H, H3), 2.27 (m, 1H, H4a), 2.19 (m, 1H, H4b), 1.43 (s, 6H, Me), 1.38 (s, 3H, Me), 1.36 (s, 3H, Me), 0.94 (s, 9H, TBS), 0.93 (s, 9H, TBS), 0.16 (s, 3H, TBS), 0.15 (s, 3H, TBS), 0.14 (s, 3H, TBS), 0.13 (s, 3H, TBS); ${ }^{13} \mathrm{C}$ NMR (75 MHz, $\left.\mathrm{CDCl}_{3}\right) \delta 175.5,109.2,109.1,77.3,76.6,76.0,71.9,66.2,65.4,55.8,46.2,26.6,26.5,26.4,26.0$ (3C), 25.9 (3C), 25.8, 25.3, 18.3, 18.1, -3.9, -4.0, -4.2, -4.7. Anal. Calcd for $\mathrm{C}_{28} \mathrm{H}_{55} \mathrm{NO}_{7} \mathrm{Si}_{2}$ : C, 58.60; $\mathrm{H}$, 9.66; N, 2.44. Found: C, 58.71; H, 9.80; N, 2.31.

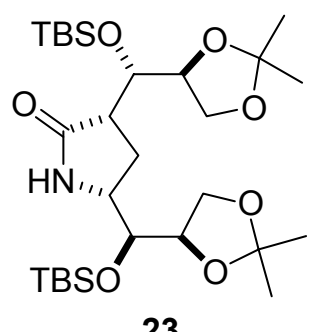

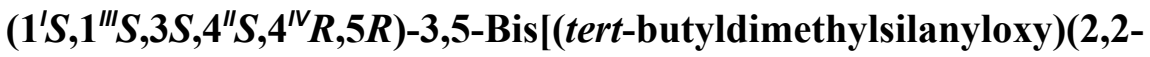

dimethyl-[1,3]dioxolan-4-yl)methyl]pyrrolidin-2-one (23). The title compound was prepared from 15 (78 $\mathrm{mg}, 0.15 \mathrm{mmol})$ in three steps according to the typical procedures described for 9. After flash chromatographic purification (EtOAc/hexanes 1:1), lactam 23 was obtained $(77 \mathrm{mg}, 90 \%)$ as a glassy solid: $[\alpha]_{\mathrm{D}}{ }^{20}-14.5\left(c 0.6, \mathrm{CHCl}_{3}\right) ;{ }^{1} \mathrm{H} \mathrm{NMR}\left(300 \mathrm{MHz}, \mathrm{CDCl}_{3}\right) \delta 5.66(\mathrm{bs}, 1 \mathrm{H}, \mathrm{NH}), 4.33$ $\left(\mathrm{dd}, J=6.8,1.2 \mathrm{~Hz}, 1 \mathrm{H}, \mathrm{H} 1^{\prime}\right), 4.06\left(\mathrm{td}, J=6.2,3.8 \mathrm{~Hz}, 1 \mathrm{H}, \mathrm{H} 4^{\prime \prime}\right), 4.02$ (q, $\left.J=6.7 \mathrm{~Hz}, 1 \mathrm{H}, \mathrm{H} 4^{\prime \prime}\right), 3.96$ (m, 2H, H5 $\left.{ }^{\prime v} \mathrm{a}, \mathrm{H} 5^{\prime v} \mathrm{~b}\right), 3.90\left(\mathrm{t}, J=7.7 \mathrm{~Hz}, 1 \mathrm{H}, \mathrm{H} 5{ }^{\prime \prime} \mathrm{a}\right), 3.71$ (t, $J=7.0 \mathrm{~Hz}, 1 \mathrm{H}, \mathrm{H} 5$ "b), 3.69 (dd, $J=8.9$, $4.0 \mathrm{~Hz}, 1 \mathrm{H}, \mathrm{H1}^{\prime \prime \prime}$ ), 3.42 (td, $\left.J=9.2,6.8 \mathrm{~Hz}, 1 \mathrm{H}, \mathrm{H} 5\right), 2.42$ (ddd, $J=10.4,9.4,1.0 \mathrm{~Hz}, 1 \mathrm{H}, \mathrm{H} 3$ ), 2.0-2.2 (m, 2H, H4a, H4b), 1.45 (s, 3H, Me), 1.43 (s, 3H, Me), 1.37 (s, 6H, Me), 0.93 (s, 9H, TBS), 0.90 (s, 9H, TBS), 0.15 (s, 6H, TBS); 0.14 (s, 3H, TBS), 0.12 (s, 3H, TBS); ${ }^{13} \mathrm{C}$ NMR (150 MHz, $\left.\mathrm{CDCl}_{3}\right) \delta 176.2$ (C2), $109.5\left(\mathrm{CMe}_{2}\right), 109.1\left(\mathrm{CMe}_{2}\right), 78.3\left(\mathrm{C}^{\prime \prime}\right), 76.5\left(\mathrm{C}^{\prime \prime \prime}\right), 76.2\left(\mathrm{C}^{\prime \prime}\right), 70.5\left(\mathrm{C} 1^{\prime}\right), 65.5\left(\mathrm{C}^{\prime \prime}\right), 64.8$ (C5"), 56.0 (C5), 44.9 (C3), 26.5 (Me), 26.3 (Me), 26.0 (3C, TBS), 25.9 (3C, TBS), 25.3 (Me), 25.2 (Me), 23.9 (C4), 18.3 (TBS), 18.1 (TBS), -3.9 (TBS), -4.0 (TBS), -4.4 (TBS), -4.9 (TBS). Anal. Calcd for $\mathrm{C}_{28} \mathrm{H}_{55} \mathrm{NO}_{7} \mathrm{Si}_{2}:$ C, 58.60; H, 9.66; N, 2.44. Found: C, 58.52; H, 9.55; N, 2.51 . 


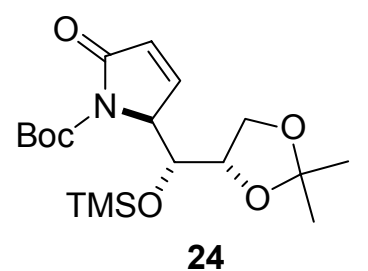

$\left(1^{\prime} R, 2 S, 4^{\prime \prime} S\right)$-2-[(2,2-Dimethyl-[1,3]dioxolan-4yl)(trimethylsilanyloxy)methyl]-5-oxo-2,5-dihydro-1 $H$-pyrrole-1-carboxylic Acid tert-Butyl Ester (24). The title compound was prepared from silyloxypyrrole 1 (700 mg, $2.35 \mathrm{mmol})$ and L-glyceraldehyde $(S)-2(367 \mathrm{mg}$, $2.82 \mathrm{mmol}$ ) in two steps, according to the typical procedures described for 4. Flash chromatographic purification (EtOAc/hexanes 6:4) afforded lactam $24(721 \mathrm{mg}, 80 \%)$ as a glassy solid: $[\alpha]_{\mathrm{D}}{ }^{20}-171.1(c$ 2.1, $\mathrm{CHCl}_{3}$ ); ${ }^{1} \mathrm{H}$ and ${ }^{13} \mathrm{C}$ NMR: see enantiomeric compound 4. Anal. Calcd for $\mathrm{C}_{18} \mathrm{H}_{31} \mathrm{NO}_{6} \mathrm{Si}$ : C, 56.08; H, 8.10; N, 3.63. Found: C, 56.00; H, 8.18; N, 3.57 .

$\left(1^{\prime} R, 1^{\prime \prime \prime} R, 4^{\prime \prime} S, 4^{\prime V} S, 5 S\right)$-3-[(2,2-Dimethyl-[1,3]dioxolan-4-yl)hydroxymethyl]-5-[(2,2-dimethyl[1,3]dioxolan-4-yl)(trimethylsilanyloxy)methyl]-2-oxo-2,5-dihydro-1 $H$-pyrrole-1-carboxylic Acid tert-Butyl Ester (25) and (1'S,1"'R,4"S, $\left.4^{\prime \prime} S, 5 S\right)$-3-[(2,2-Dimethyl-[1,3]dioxolan-4-yl)hydroxymethyl]5-[(2,2-dimethyl-[1,3]dioxolan-4-yl)(trimethylsilanyloxy)methyl]-2-oxo-2,5-dihydro-1H-pyrrole-1carboxylic Acid tert-Butyl Ester (26). The title compounds were prepared from lactam 24 (360 mg, $0.93 \mathrm{mmol})$ and L-glyceraldehyde $(S)-2(242 \mathrm{mg}, 1.86 \mathrm{mmol})$ according to the typical procedure described for 5 and 6. After flash chromatographic purification (EtOAc/hexanes 35:65) adducts 25 (297 $\mathrm{mg}, 62 \%)$ and $\mathbf{2 6}(86 \mathrm{mg}, 18 \%)$ were recovered in a $80 \%$ global yield.

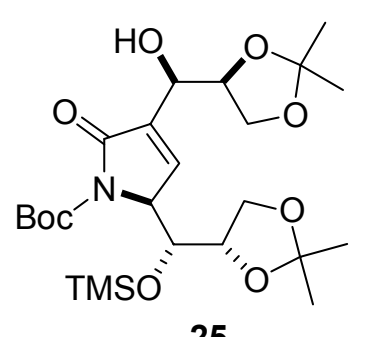

Compound 25: a glassy solid, $[\alpha]_{\mathrm{D}}{ }^{20}-101.7$ (c 1.4, $\left.\mathrm{CHCl}_{3}\right) ;{ }^{1} \mathrm{H}$ and ${ }^{13} \mathrm{C}$ NMR: see enantiomer 5. Anal. Calcd for $\mathrm{C}_{24} \mathrm{H}_{41} \mathrm{NO}_{9} \mathrm{Si}: \mathrm{C}, 55.90 ; \mathrm{H}, 8.01 ; \mathrm{N}$, 2.72. Found: C, 55.83; H, 7.92; N, 2.88 .

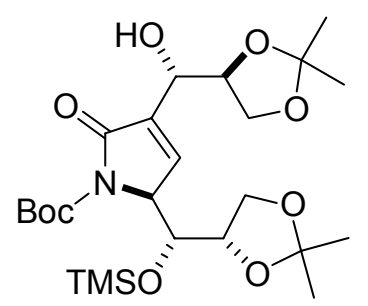

Compound 26: a glassy solid, ${ }^{1} \mathrm{H}$ and ${ }^{13} \mathrm{C}$ NMR: see enantiomer 6. Anal. Calcd for $\mathrm{C}_{24} \mathrm{H}_{41} \mathrm{NO}_{9} \mathrm{Si}$ : C, 55.90; H, 8.01; N, 2.72. Found: C, 56.01; H, 8.12; N, 2.64 .

$\left(1^{\prime} R, 1^{\prime \prime \prime} R, 4^{\prime \prime} R, 4^{\prime \prime} S, 5 S\right)$-3-[(2,2-Dimethyl-[1,3]dioxolan-4-yl)hydroxymethyl]-5-[(2,2-dimethyl[1,3]dioxolan-4-yl)(trimethylsilanyloxy)methyl]-2-oxo-2,5-dihydro-1 $H$-pyrrole-1-carboxylic Acid tert-Butyl Ester (27) and $\quad\left(1^{\prime} S, 1^{\prime \prime \prime} R, 4^{\prime \prime} R, 4^{\prime \prime} S, 5 S\right)$-3-[(2,2-Dimethyl-[1,3]dioxolan-4yl)hydroxymethyl]-5-[(2,2-dimethyl-[1,3]dioxolan-4-yl)(trimethylsilanyloxy)methyl]-2-oxo-2,5dihydro-1 H-pyrrole-1-carboxylic Acid tert-Butyl Ester (28). The title compounds were prepared from unsaturated lactam 24 (360 mg, $0.93 \mathrm{mmol})$ and D-glyceraldehyde $(R)-2(242 \mathrm{mg}, 1.86 \mathrm{mmol})$ 
according to the typical procedure described for $\mathbf{5}$ and 6. Flash chromatographic purification (EtOAc/hexanes 35:65) of the residue furnished $67 \mathrm{mg}$ of compound $27(14 \%)$<smiles></smiles>

27 and $302 \mathrm{mg}$ of $\mathbf{2 8}(63 \%)$.

Compound 27: a colorless oil, $[\alpha]_{\mathrm{D}}{ }^{20}-67.9\left(c\right.$ 1.6, $\left.\mathrm{CHCl}_{3}\right) ;{ }^{1} \mathrm{H}$ NMR: see enantiomeric counterpart 15. Anal. Calcd for $\mathrm{C}_{24} \mathrm{H}_{41} \mathrm{NO}_{9} \mathrm{Si}$ : C, 55.90; H, 8.01; N, 2.72. Found: C, 55.76; H, 8.13; N, 2.78.<smiles>CO[C@H]([C@H]1COC(C)(C)O1)[C@H]1C=C([C@H](O)[C@H]2COC(C)(C)O2)C(=O)N1C(=O)OC(C)(C)C</smiles>

28

Compound 28: a colorless oil, $[\alpha]_{\mathrm{D}}{ }^{20}-76.2\left(\mathrm{c} 0.9, \mathrm{CHCl}_{3}\right) ;{ }^{1} \mathrm{H}$ and ${ }^{13} \mathrm{C}$ NMR: see enantiomeric counterpart 14. Anal. Calcd for $\mathrm{C}_{24} \mathrm{H}_{41} \mathrm{NO}_{9} \mathrm{Si}$ : C, 55.90; H, 8.01; N, 2.72. Found: C, 55.99; H, 7.89; N, 2.84.

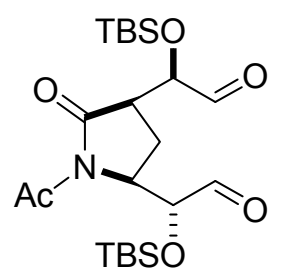

29

\section{$\left(2 R, 2^{\prime} R, 2^{\prime \prime} S, 4^{\prime \prime} R\right)-2,2^{\prime}$-(1-Acetyl-5-oxopyrrolidine-2,4-diyl)bis(tert-}

butyldimethylsilanyloxyacetaldehyde) (29). The title compound was prepared from lactam 25 (297 $\mathrm{mg}, 0.58 \mathrm{mmol})$ in five steps, according to the typical procedures described for 11. Flash chromatographic purification (EtOAc/hexanes 6:4) of the residue afforded aldehyde $29(180 \mathrm{mg}, 66 \%)$ as a colorless oil: $[\alpha]_{\mathrm{D}}^{20}+54.3(c 0.6$, $\left.\mathrm{CHCl}_{3}\right) ;{ }^{1} \mathrm{H}$ and ${ }^{13} \mathrm{C}$ NMR: see enantiomer 11. Anal. Calcd for $\mathrm{C}_{22} \mathrm{H}_{41} \mathrm{NO}_{6} \mathrm{Si}_{2}: \mathrm{C}, 56.01 ; \mathrm{H}, 8.76 ; \mathrm{N}$, 2.97. Found: C, 56.10; H, 8.87; N, 2.82.

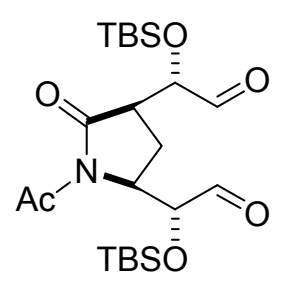

30

\section{$\left(2 R, 2^{\prime} S, 2^{\prime \prime} S, 4^{\prime \prime} R\right)-2,2^{\prime}$-(1-Acetyl-5-oxopyrrolidine-2,4-diyl)bis(tert-}

butyldimethylsilanyloxyacetaldehyde) (30). The title compound was prepared from lactam 28 (302 $\mathrm{mg}, 0.59 \mathrm{mmol})$ in five steps, according to the typical procedures described for 11. Flash chromatographic purification (EtOAc/hexanes 6:4) of the residue afforded aldehyde $30(180 \mathrm{mg}, 65 \%)$ as a colorless oil: $[\alpha]_{\mathrm{D}}{ }^{20}-50.9(c$ 1.0,

$\left.\mathrm{CHCl}_{3}\right) ;{ }^{1} \mathrm{H}$ and ${ }^{13} \mathrm{C}$ NMR: see enantiomer 19. Anal. Calcd for $\mathrm{C}_{22} \mathrm{H}_{41} \mathrm{NO}_{6} \mathrm{Si}_{2}: \mathrm{C}, 56.01 ; \mathrm{H}, 8.76 ; \mathrm{N}$, 2.97. Found: C, 56.10; H, 8.87; N, 2.82 . 


\section{References}

(1) Rassu, G.; Zanardi, F.; Battistini, L.; Gaetani, E.; Casiraghi, G. J. Med. Chem. 1997, 40, 168-180.

(2) Zanardi, F.; Battistini, L.; Rassu, G.; Auzzas, L.; Pinna, L.; Marzocchi, L.; Acquotti, D.; Casiraghi, G. J. Org. Chem. 2000, 65, 2048-2064.

(3) Hubschwerlen, C.; Specklin, J.-L.; Higelin, J. Org. Synth. 1995, 72, 1-5.

(4) The experimental procedure and spectroscopic data for compound $\mathbf{3}$ have been reported before (see Ref. 10 in the text). However, they are included again here since the original experimental procedure has been optimized. 
${ }^{1} \mathrm{H}$ NMR spectrum $\left(600 \mathrm{MHz}, \mathrm{CDCl}_{3}\right)$ of lactam $\mathbf{5}$

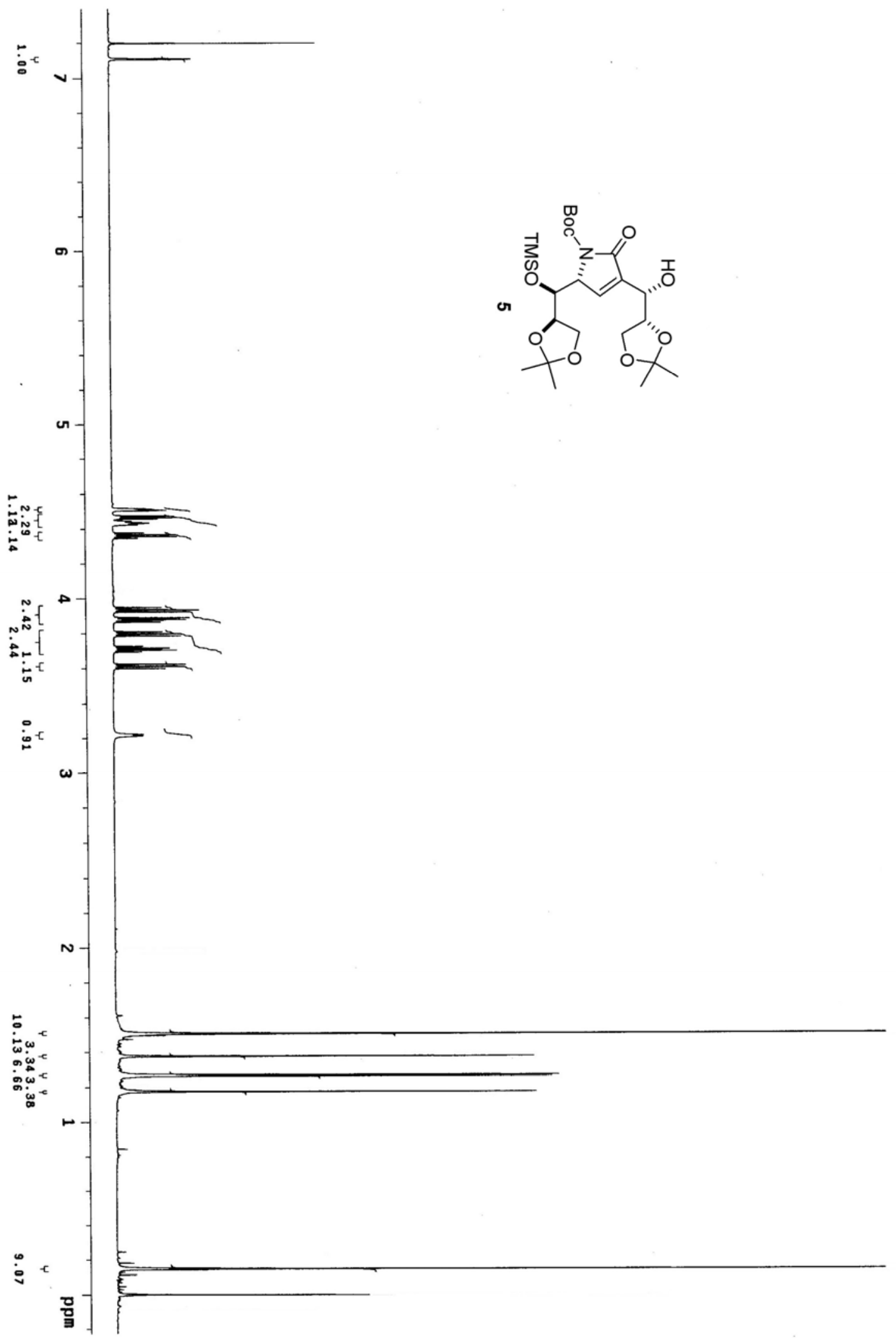


${ }^{1} \mathrm{H}$ NMR spectrum $\left(600 \mathrm{MHz}, \mathrm{CDCl}_{3}\right)$ of bicycle 12

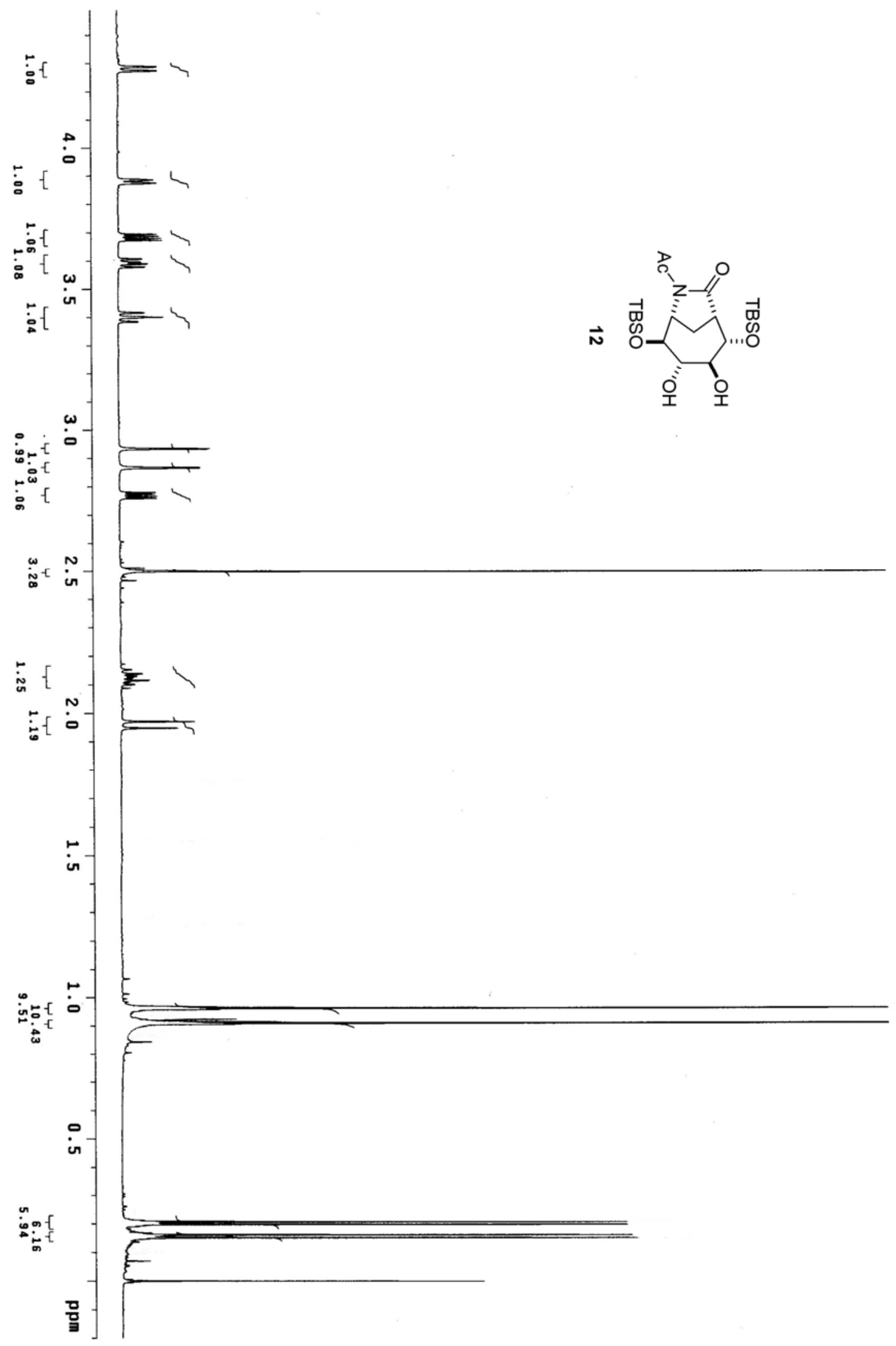


${ }^{1} \mathrm{H}$ NMR spectrum $\left(600 \mathrm{MHz}, \mathrm{D}_{2} \mathrm{O}\right)$ of amino acid $\mathbf{1 3}$

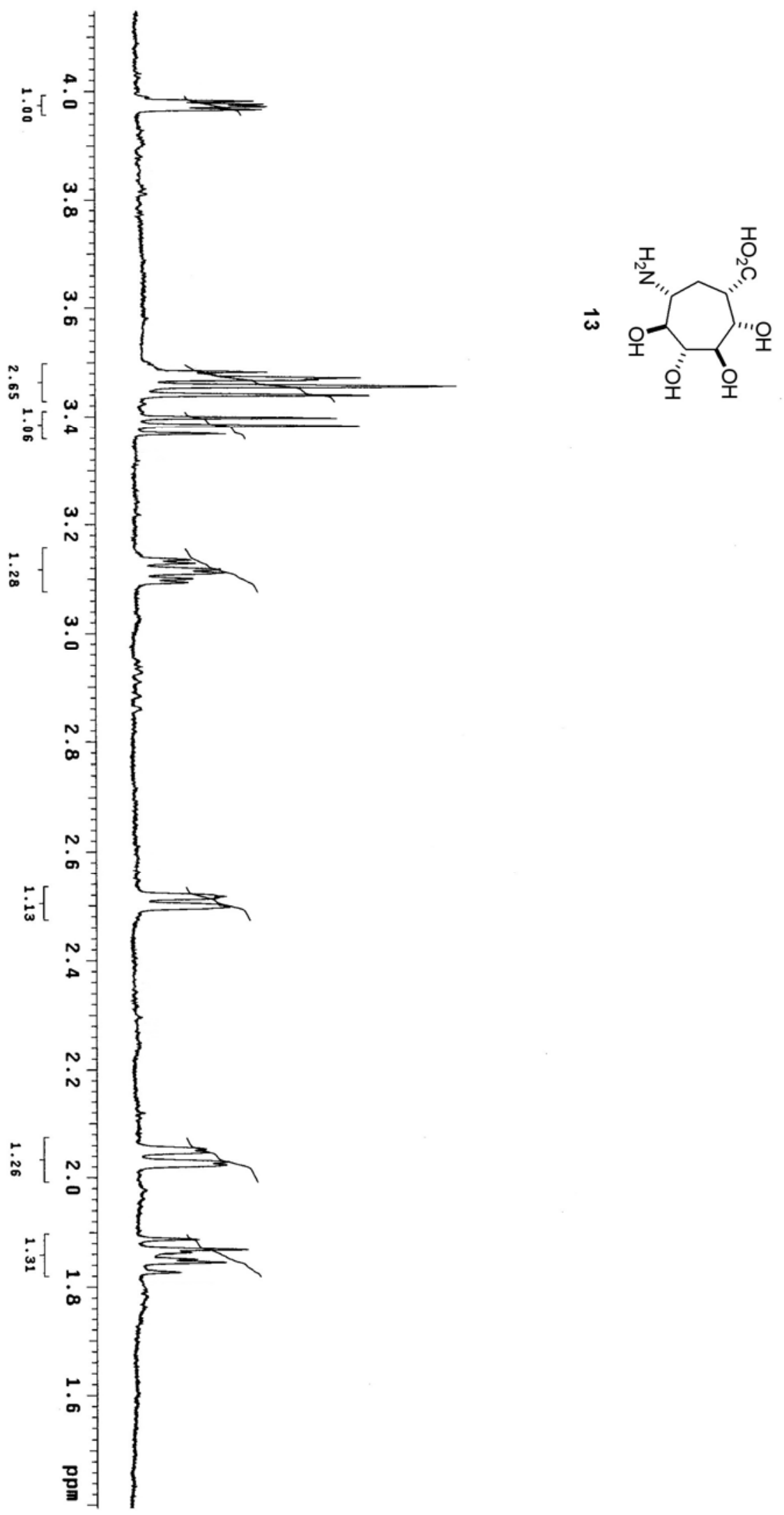


${ }^{1} \mathrm{H}$ NMR spectrum $\left(600 \mathrm{MHz}, \mathrm{CDCl}_{3}\right)$ of lactam 14

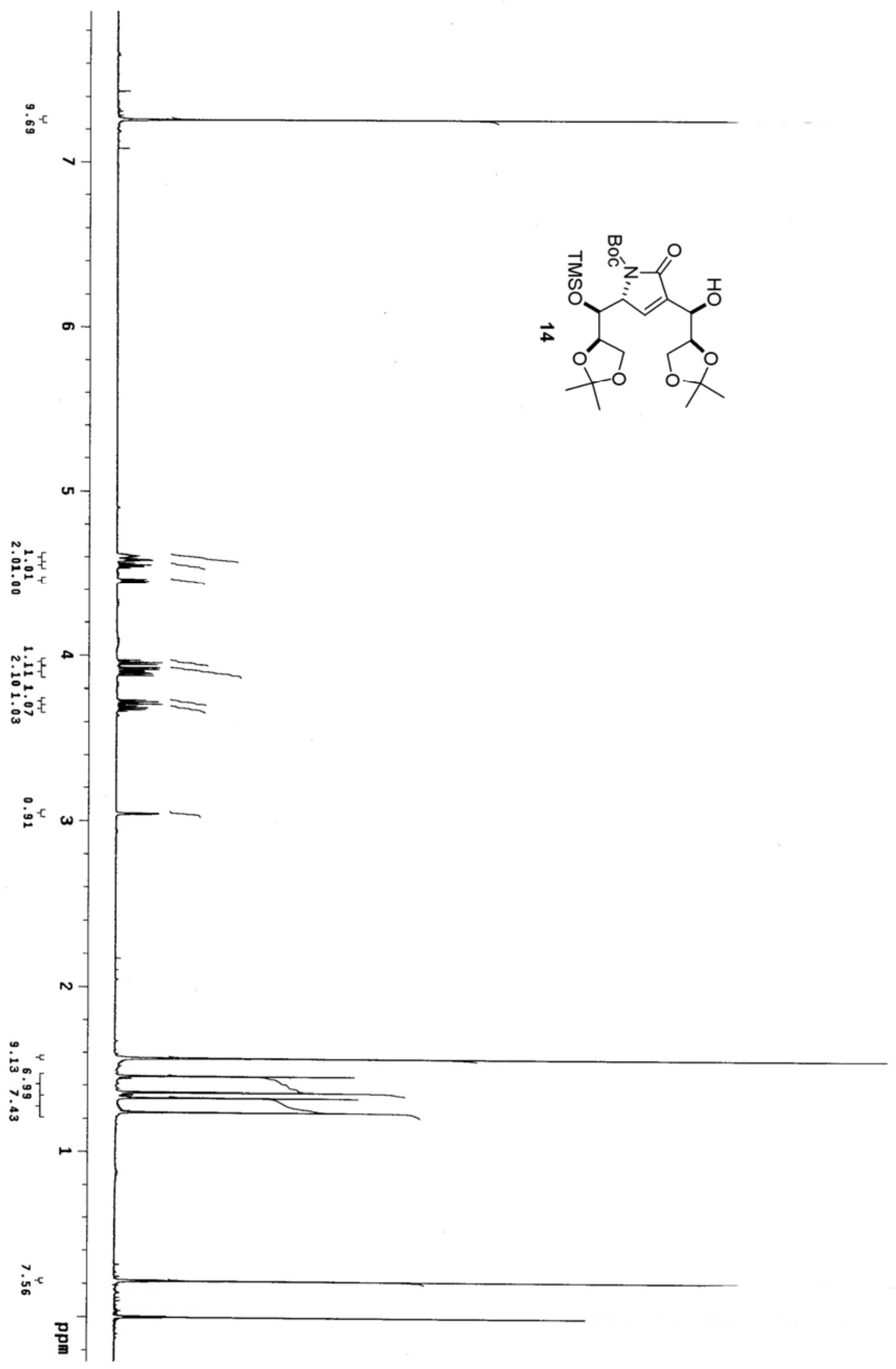


${ }^{1} \mathrm{H}$ NMR spectrum $\left(600 \mathrm{MHz}, \mathrm{CDCl}_{3}\right)$ of bicycle $\mathbf{2 0}$

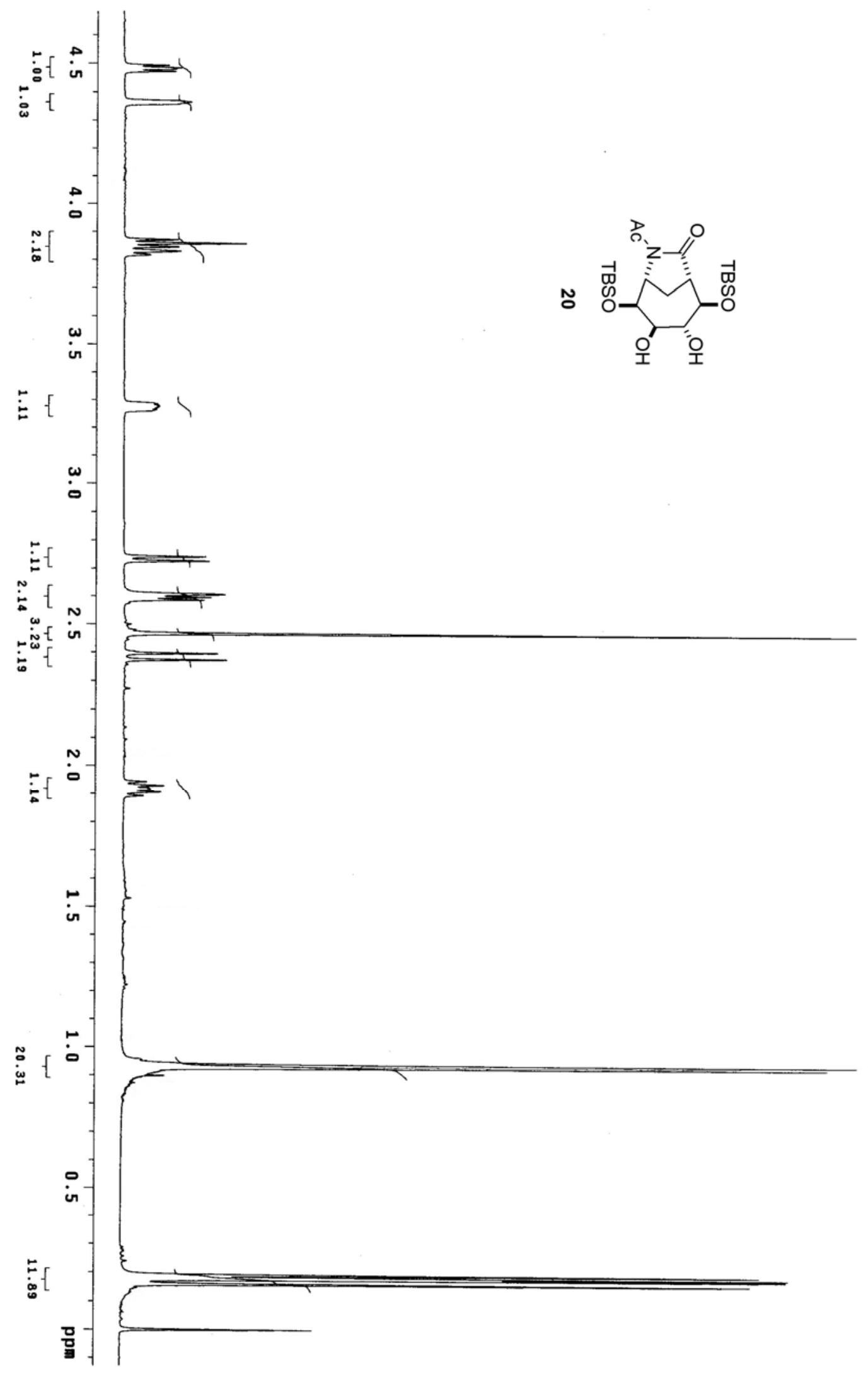


${ }^{1} \mathrm{H}$ NMR spectrum $\left(600 \mathrm{MHz}, \mathrm{D}_{2} \mathrm{O}\right)$ of amino acid 21
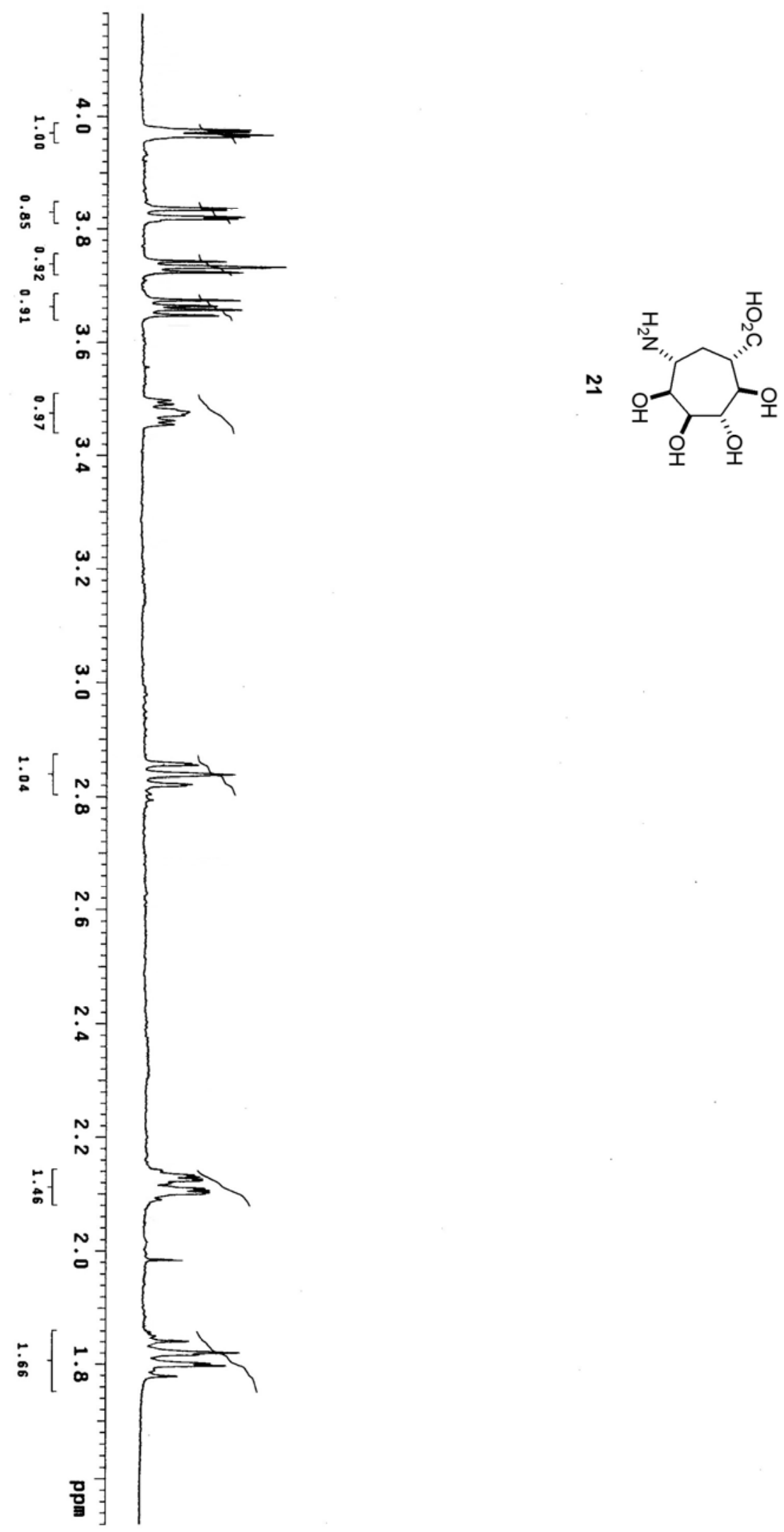